\title{
A CONTINUUM $X$ WHICH IS A RETRACT OF $C(X)$ BUT NOT OF $2^{X}$
}

\author{
ALEJANDRO ILLANES
}

\begin{abstract}
We give an example of a continuum $X$ which is a retract of $C(X)$
\end{abstract} but not of $2^{X}$.

1. Introduction. A continuum is a compact connected metric space. The hyperspaces of a continuum $X$ are the spaces $2^{X}=\{A \in X: A$ is closed and nonempty $\}$ and $C(X)=\left\{A \in 2^{X}: A\right.$ is connected $\}$, each with the Hausdorff metric. We identify $X$ with the subspace $\left\{\{x\} \in 2^{X}: x \in X\right\}$ of $2^{X}$ (and $C(X)$ ). If $p$ is a point of $X$, we define $C_{p}(X)=\{A \in C(X): p \in A\}$. A map is a continuous function. We say that a map $\lambda: X \times X \rightarrow X$ is a mean if $\lambda(x, y)=\lambda(y, x)$ for all $x, y \in X$ and $\lambda(x, x)=x$ for all $x \in X$.

It is known [4, Theorem 6.4] that if $X$ is a locally connected continuum, then the following assertions are equivalent:

(a) $X$ is an absolute retract.

(b) $X$ is a retract of $C(X)$.

(c) $X$ is a retract of $2^{X}$.

This result has motivated the question asked by S. B. Nadler, Jr. [4, question 6.28], of whether for every continuum, (b) and (c) are equivalent. The aim of this paper is to show a continuum $X$ for which (b) holds while (c) does not hold (in fact $X$ does not admit a mean). Other interesting examples concerning retractions between hyperspaces can be found in $[\mathbf{1}, \mathbf{2}$, and $\mathbf{3}]$.

I express my thanks to Professor D. Curtis for his suggestions to simplify the example and its construction.

2. Construction of the example $X$. Consider the triod $T=[-1,1] \times\{0\} \cup$ $\{0\} \times[0,1] \subset \mathbf{R}^{2}$, the convergent sequence $J=\{1 / n: n=1,2, \ldots\} \cup\{0\} \subset \mathbf{R}$ and the cone Cone $(T \times J)=\{(s, t, j): 0 \leq s \leq 1, t \in T, j \in J\}$ with the cone point $p=(0, t, j)$.

Let $\tau \subset 2^{T}$ denote the collection of all three-element subsets $\{x, y, z\}$ of $T$ such that one of the points $x, y$, or $z$ is between (i.e. separates) the other two points in $T$. Use the natural convex structure on $T$ to define $u x+v y$ in $T$, for all $x, y \in T$, $u, v \geq 0$ with $u+v=1$. For each $\sigma=\{x, y, z\} \in \tau$ with $z$ between $x$ and $y$, define the triod $T_{\sigma} \subset \operatorname{Cone}(T \times\{0\})$ as follows:

$$
\begin{aligned}
T_{\sigma}= & \{(s, z, 0): 0 \leq s \leq 1 / 2\} \cup\{(s,(2 s-1) x+(2-2 s) z, 0): 1 / 2 \leq s \leq 1\} \\
& \cup\{(s,(2 s-1) y+(2-2 s) z, 0): 1 / 2 \leq s \leq 1\} .
\end{aligned}
$$

Let $\left\{\sigma_{n}: n=1,2, \ldots\right\}$ be a dense sequence in $\tau$, and for each $n$ define the triod $T_{n} \subset \operatorname{Cone}(T \times\{1 / n\})$ by $T_{n}=\left\{(s, t, 1 / n):(s, t, 0) \in T_{\sigma_{n}}\right\}$. Then define

Received by the editors November 15, 1985 and, in revised form, February 14, 1986.

1980 Mathematics Subject Classification (1985 Revision). Primary 54B20; Secondary 54C15.

Key words and phrases. Continuum, hyperspace, retraction. 
$X \subset \operatorname{Cone}(T \times J)$ by $X=\bigcup\left\{T_{n}: n=1,2, \ldots\right\} \cup \operatorname{Cone}(T \times\{0\})$. (Of course, $X$ could be imbedded in $\mathbf{R}^{3}$, but this would unnecessarily complicate its description.)

3. $X$ does not admit a mean. Suppose there exists a mean $\lambda: X \times X \rightarrow X$. Let $d$ be the path-length metric on the triod $T$. Choose $\varepsilon>0$ such that, for every pair $(s, t, j),\left(s, t^{\prime}, j\right)$ in $X$ with $s \geq 1 / 2$ and $d\left(t, t^{\prime}\right) \leq 2 \varepsilon, \lambda\left((s, t, j),\left(s, t^{\prime}, j\right)\right) \neq p$, the cone point. For convenience, identify $T$ with the subset $\{1\} \times T \times\{0\}$ of Cone $(T \times\{0\})$. Thus $T \subset X$.

Claim. For each $x, y \in T$ with $d(x, y) \leq \varepsilon, \lambda(x, y) \in\{x, y\}$. Assuming $x \neq y$, consider any $z \in T$ between $x$ and $y$. Choose a subsequence $\left\{\sigma_{n_{i}}\right\}$ such that $\sigma_{n_{i}} \rightarrow \sigma=\{x, y, z\}$. Then $T_{n_{i}} \rightarrow T_{\sigma}$. Let $e_{n_{i}}, f_{n_{i}}$ denote the endpoints of $T_{n_{i}}$ different from $p$, labeled so that $e_{n_{i}} \rightarrow x$ and $f_{n_{i}} \rightarrow y$. By the choice of $\varepsilon$, we must have $\lambda\left(e_{n_{i}}, f_{n_{i}}\right) \in T_{n_{i}}$ for all $n_{i}$ sufficiently large. Thus $\lambda(x, y) \in T_{\sigma}$. Since this holds for any $\sigma=\{x, y, z\}$ with $z$ between $x$ and $y$, it follows that $\lambda(x, y) \in\{x, y\}$.

Now consider the points $(0,0),( \pm \varepsilon, 0),(0, \varepsilon)$ of $T$. We may suppose by the claim that $\lambda((0,0),(\varepsilon, 0))=(\varepsilon, 0)$. By continuity of $\lambda$, this implies that $\lambda((0, \varepsilon),(0,0))=$ $(0,0)$, and that $\lambda((-\varepsilon, 0),(0,0))=(0,0)$. But the latter implies that $\lambda((0,0),(0, \varepsilon))$ $=(0, \varepsilon)$, contradicting the hypothesis that $\lambda$ is a mean.

4. $C(X)$ retracts to $X$. Let $l: X \rightarrow[0,1]$ be the projection map defined by $l(s, t, j)=s$. Let $\mathcal{L} \subset C(\operatorname{Cone}(T \times\{0\}))$ be the closed subhyperspace defined by $\mathcal{L}=\left\{M: M=\lim M_{i}\right.$, for some sequence $\left\{M_{i}\right\}$ in $C(X)$ such that $M_{i} \subset T_{n_{i}}$, with $\left.n_{i} \rightarrow \infty\right\}$. Then for each $M \in \mathcal{L}$, there exists a unique $x \in M$ such that $l(x)=\inf l(M)$.

Let $r: C(\operatorname{Cone}(T \times\{0\})) \rightarrow \operatorname{Cone}(T \times\{0\})$ be any retraction satisfying the conditions:

(1) $r(M)=p$ if $p \in M$; and

(2) for each $M \in \mathcal{L}, r(M) \in M$ is such that $l(r(M))=\inf l(M)$.

Since Cone $(T \times\{0\})$ is an AR, such a retraction may be constructed by first defining $r \mid \operatorname{Cone}(T \times\{0\})=$ id, then defining $r$ over $C_{p}(\operatorname{Cone}(T \times\{0\})) \cup \mathcal{L}$ by conditions (1) and (2), and finally, extending $r$ over the entire hyperspace $C($ Cone $(T \times\{0\})$.

Then the desired retraction $r: C(X) \rightarrow X$ is obtained as the extension of the above $r$ satisfying the conditions:

$\left(1^{*}\right) r(M)=p$ if $p \in M ;$ and

$\left(2^{*}\right)$ for each $M \in C(X)$ with $M \subset T n$ for some $n, r(M) \in M$ is such that $l(r(M))=\inf l(M)$.

\section{REFERENCES}

1. D. W. Curtis, A hyperspace retraction theorem for a class of half-line compactifications, preprint.

2. J. T. Goodykoontz, Jr., $C(X)$ is not necessarily a retract of $2^{X}$, Proc. Amer. Math. Soc. 67 (1977), 177-178.

3. J. T. Goodykoontz, Jr., A nonlocally connected continuum $X$ such that $C(X)$ is a retract of $2^{X}$, Proc. Amer. Math. Soc. 91 (1984), 319-322.

4. S. B. Nadler, Jr., Hyperspaces of sets, Dekker, New York, 1978.

Instituto de Matematicas, Circuito Exterior, Ciudad Universitaria, MEXICO, D.F. C.P. 04510 